\title{
Neuroscience-Based Formulation and Treatment for Early-Onset Bipolar Disorder: a Paradigm Shift
}

\section{Mani Pavuluri, MD, PhD}

\section{Address}

Pediatric Mood Disorders Program and Pediatric Brain Research and Intervention Center, Department of Psychiatry, University of Illinois at Chicago, M/C 747, Chicago, IL 60608, USA

Email: Mpavuluri@psych.uic.edu

Published online: 10 July 2015

(C) Springer International Publishing AG 2015

This article is part of the Topical Collection on Mood Disorders

Keywords Brain - Bipolar disorder · Treatment · RDoC · Cognitive · Affective · Domains

\section{Opinion statement}

Treating pediatric bipolar disorder (BD) requires new knowledge based on emerging and consistent neuroscience findings. It entails recognition and addressing the deficits in eight domains such as emotion processing, executive function, attention, impulse control, working memory, reward and risk response, perspective taking, and, finally, the interface of emotion and cognition. Skillful integration of four components: (A) assessment (disorder and domain functions), (B) educating the families that it is early-onset brain dysfunction based on what we now know through research, $(C)$ chemotherapy with the hope of reversing the brain dysfunction, and (D) addressing the individual dynamics specific to the family and through neuroscience informed psychological treatment such as RAINBOW therapy. Imaging findings in pediatric BD offered four major contributions: (1) prefrontal cortex regions improve in function with some pharmacological agents, while amygdala in the subcortical region does not reach normality till after longerterm treatment; (2) various medications differentially engage different brain circuitry regions, supporting why combined therapy is required in severely ill BD; (3) there is invariable interlink between affective and cognitive domain dysfunctions in pediatric $\mathrm{BD}$, by the virtue of early-onset illness diathesis; and (4) consistent and strong evidence that negative stimuli impact both affect regulation and cognitive function argues towards not using negative "punishment" in psychological treatment, thereby leading to the construction and completion of the randomized controlled trial of RAINBOW therapy or child- and family-focused cognitive behavior therapy. Ultimately, translating the new research findings to clinically ill 
population and educating researchers on clinical challenges are both critical steps towards a determined advancement with a singular focus to help children and their families.

\section{Introduction}

Understanding the underpinnings of what to treat and how to treat a child, an adolescent, and their family with bipolar disorder (BD) is fully realized only when the treating clinician develops a comfort zone with the integration of the emerging scientific knowledge with clinical experience. Such blend in skills paired with updated knowledge on neuroscience offers the ability to effectively treat the illness that presents with high comorbidity and complex multi-domain dysfunction.
BD does not pose a unique example in pediatric psychiatry, but serves as a prototype that calls for precision in treating multiple problems in a developing child. The approach to treating $\mathrm{BD}$ is presented in four parts (the A, B, C, D model): assessment (A), brain and behavioral function informing treatment (B), chemotherapy or pharmacotherapy (C), and delivery of treatment through addressing the dynamics of a given individual family (D) $[1 \bullet]$.

\section{A. Assessment}

Bipolar disorder (BD) is a specific psychiatric symptom cluster with high degree of variability in its presentation due to the multiple combinations of symptoms and the duration of the episodes $[2,3 \bullet \bullet, 4]$. The defining symptoms of elated or irritable mood, inflated self-esteem or grandiosity, hypersexuality and/or other risk-taking behaviors, flight of ideas and pressure of speech, high energy and increased goal-directed behavior that signify exalted affective state and heightened motor activity may not all be present at one point in time, in every affected individual that is manic or hypomanic [5]. The presence of a minimum of four of these symptoms (three, if accompanied by abnormal elation) in children, lasting for a week or more, counts as an episode of mania according to Diagnostic and Statistical Manual of Mental Disorders, Fifth Edition (DSM 5) [6]. These episodes manifest as a qualitative change from the children's normative baseline. The presence of these symptoms of activation without functional impairment and for a shorter and minimum period of 4 days amounts to hypomanic episode. At least one major depressive episode alternating with hypomania is termed BD type 2, and mania with or without major depressive episode is termed as BD type 1 . The fluctuating mood states that are episodic with waxing and waning quality over and above the chronic dysfunction favor the diagnosis of $\mathrm{BD}$. If the symptoms are in the realm of bipolar diathesis, but do not meet either the number or duration criteria, the condition is termed specified bipolar and related disorder (or unspecified if unable to specify the exact symptoms or duration). Diagnostic categories are the mainstay in communicating the core clusters of symptoms and pattern.

Frequently, residual affect dysregulation and high degree of emotional reactivity to negative stimuli persists in between the episodes [7]. Parents also report chronic inter-episodic morbidity that is analogous to difficult temperament from early childhood [8]. It is hard to differentiate emotional reactivity from disruptive mood dysregulation disorder (DMDD) described in DSM 5, or 
a variant of oppositional defiant disorder (ODD) under consideration as a subtype of ODD in ICD 11 [9] where externalizing behavior, affective intensity, and autonomic hyperarousal are predominant [10]. Symptoms of DMDD may underlie BD, but once BD manifests as a full-blown disorder, DMDD is not recognized as a coexisting or underlying disorder. Furthermore, inattention, impulsivity, and overactivity are common in BD and may persist in interepisodic phase [11]. Unless parents attest that attention deficit hyperactivity disorder (ADHD) with inattention, impulsivity, and overactivity preceded BD, these symptoms could be construed as neurocognitive sequel secondary to $\mathrm{BD}$ [12-14], similar to those in adult BD [15]. Therefore, ADHD is considered as comorbid disorder only if it preceded the onset of BD. The studies that reported high rates of comorbidity at $97 \%$ [16] measured the cross-sectional symptoms of ADHD present during the manic episode. From a treatment perspective, it appears that there is no optimal form to report the cognitive symptoms that resemble ADHD if they are present between the episodes of hypo/mania that need to be addressed without word wars across diagnosticians.

\section{B. Brain and behavioral function informing treatment}

In addition to the clinical diagnosis, another tier of assessment entails capturing the emotional and cognitive domain difficulties that are typical in early-onset BD (Fig. 1).

\begin{tabular}{|c|c|c|}
\hline $\begin{array}{l}\text { Impaired Emotion } \\
\text { Processing } \\
\text { Excessive reactivity to } \\
\text { negative emotions } \\
\text { Impaired face emotion } \\
\text { recognition } \\
\text { Missing subtle cues }\end{array}$ & $\begin{array}{l}\text { Executive Dysfunction } \\
\text { Cognitive inflexibility } \\
\text { Poor problem solving } \\
\text { Poor forward planning and } \\
\text { shifting } \\
\text { Story telling and detailed } \\
\text { verbal elaboration-poor }\end{array}$ & $\begin{array}{l}\text { Impulsivity } \\
\text { Impaired ability to control } \\
\text { responses, and accurately } \\
\text { time the responses }\end{array}$ \\
\hline $\begin{array}{l}\text { Reward and risk processing } \\
\text { Excitability with rewards } \\
\text { Frustration with losses } \\
\text { Inability to wait for reward }\end{array}$ & Bipolar Disorder & $\begin{array}{l}\text { Inattention } \\
\text { Sustained attention } \\
\text { problems } \\
\text { Biased attention to } \\
\text { negative cues }\end{array}$ \\
\hline $\begin{array}{l}\text { Perspective taking deficits } \\
\text { Inability to grasp hints, } \\
\text { especially in the context of } \\
\text { negative stories }\end{array}$ & $\begin{array}{l}\text { Interface of Emotion and } \\
\text { Cognition: Impairment } \\
\text { Working memory, impulse } \\
\text { control, contingency } \\
\text { response or attention are } \\
\text { all affected in the context } \\
\text { of intense emotions, } \\
\text { especially the negative } \\
\text { emotions }\end{array}$ & $\begin{array}{l}\text { Working memory deficits } \\
\text { Cannot remember numbers } \\
\text { or words, especially if } \\
\text { complex or multiple steps } \\
\text { are involved in learning, } \\
\text { remembering verbal } \\
\text { information, math or } \\
\text { reading material }\end{array}$ \\
\hline
\end{tabular}

Fig. 1. Bipolar disorder and domain dysfunction: targets for intervention. 
Cognitive problems such as attention, working memory, verbal memory, and executive function and emotion processing difficulties persist in between the illness episodes; worsen with age, especially if untreated; and lead to poor academic and psychosocial function. We showed that academic functioning worsened over time with math difficulties increasing from 32 to $62 \%$ particularly in those with lower verbal memory [14], whereas reading difficulties were associated with attentional difficulties [17]. An additional challenge in this population is that the affect dysregulation that is central to the BD hampers cognitive operations and vice versa. The illness diathesis is likely to hamper natural brain maturation unless the impairment in multi-domain functionality is addressed in a timely fashion. Therefore, detailed clinical inquiry helps to educate our teachers, families, and patients on how to address cognitive impairments in the early years.

The last decade of scientific findings explain the multi-domain brain dysfunction that needs to be considered in treatment (Fig. 1). All results reported from the studies here were in BD relative to healthy controls unless specified otherwise.

\section{Emotion}

\section{A.I. Behavioral findings}

\section{A.II. Clinical translation based on behavioral findings}

Patients in euthymic state with BD showed impairment in detecting subtle emotions, signifying a trait deficit [18]. Furthermore, patients with BD

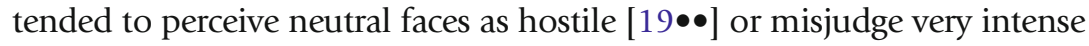
happy or sad emotions as mild [20]. Emotion differentiation is more prominent in acutely ill and those with earlier onset of illness than euthymic or later onset samples [21].

These findings may mean that patients with BD miss the subtle interpersonal emotional cues or misjudge intense emotions or react to perceived negativity in their environment that becomes an external stressor. A combination of these deficits will likely perpetuate interpersonal conflict and generate stress. Stress can trigger and maintain rapid mood fluctuations (rapid cycling). Therefore, it becomes important that patients read the environmental cues carefully and accurately recognize the emotions of those around them. That would release them from the affective hold of negative perception and can facilitate the ability to focus on the cognitive tasks at hand as explained further through fMRI studies [22•].

\section{B.I. Emotion processing circuitry}

Various types of negative stimuli such as angry faces or scathing words, more than positive faces or words, have consistently elicited fronto-limbic abnormalities in BD. The prefrontal cortical (PFC) that is emotion control region called the ventrolateral prefrontal cortex (VLPFC) along with the dorsolateral PFC (DLPFC) modulates overactive amygdala that receives and reacts to the external stimuli and could be influenced by the internal mood state. There is consistent evidence to show a total "shut down" or no activity 


\section{B.II. Clinical translation based on brain function}

in right VLPFC in patient during mania and inefficient overactivation of this region during effortful control during less severe mood episodes, i.e., hypomania. It is not conclusive if the abnormalities are primarily cortical or subcortical and are likely to be present at the brain-wide system level [2325]. Animal studies showed strong connectivity between VLPFC, amygdala, anterior cingulate cortex (ACC), and insula [1 $\bullet$ with the pregenual ACC acting as intermediary compensatory emotional control region. The insula is posteriorly contiguous with VLPFC and engages in processing negative emotions and is also responsible for arousal on processing sensorimotor information [26].

The prefrontal cortex, specifically the right VLPFC in conjunction with the DLPFC, shows underactivity, and the amygdala shows overactivity in response to angry faces in $\mathrm{BD}$ type I suggesting that these patients are reactive to negative stimuli. While not directly tested, this finding led me often to suggest environmental manipulation where parents could choose compassionate teacher vs. the strict teacher (with assumption that either teacher could provide structure).

Another critical finding was that amygdala activity was lower when labeling an emotion (be it happy or angry face) compared to attending to non-emotional problem solving (e.g., estimating age of the face) under emotional challenge [27] in BD. So consciously focusing on labeling a facial emotion may improve PFC regulation of the amygdala in pediatric BD. Conversely, visual processing and labeling of facial affect when attention is undivided may be less taxing to the PFC in regulating amygdala activity in BD compared to when emotional stimuli are presented unexpectedly or as distractors. Therefore, a therapeutic strategy is to teach children to recognize, note, and take perspective of others' facial emotions through conscious observation. That could protect them from avoiding automatic excessive reactivity to incidental or perceived negative emotions.

\section{Executive function and interlinked multi-domain problems}

\section{A.I. Behavioral findings}

Executive function encompasses higher cognitive operations that include information processing, flexibility, problem solving, organization, and emotion regulation. We showed that executive dysfunction progressed with age [14] and did not improve with optimal mood stabilization [14, 25, 28] regardless of whether patients had comorbid ADHD or were ill or euthymic $[12,25]$. Cognitive flexibility, adaptation to changing risks and rewards, frustration tolerance to negative consequences $[19 \bullet \bullet, 29]$, error monitoring with feedback [29, 30], forward planning with changing rules [31], and processing speed [13] were shown to be significantly impaired in patients with BD. Finally, meta-analysis of all the aforementioned studies highlighted deficits in planning, inhibition, set-shifting, full-scale intelligence quotient (IQ), and academic functioning [32]. Furthermore, negative emotional material was found to proactively interfere with the executive function of language involving encoding and recall of microstructure or 


\section{A.II. Clinical translation based on behavioral findings}

detail of the past events [33]. The level of depressive symptoms appeared to adversely impact recall of microstructure, but not macrostructure or the gist of the story in language use and reproduction, especially when recounting negatively valanced stories.

These findings show that patients with $\mathrm{BD}$ are not able to adapt to changing environmental cues as easily, have difficulties in planning ahead, resist transitions from one activity to the other (especially if it is shifting from activities that offer immediate gratification like video gaming to studying), become reactive under stress, and are slow in

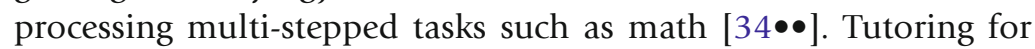
multi-stepped learning, reduced homework to decrease cognitive burden and increased motivation through success, increased test taking duration, help with problem solving through preparation and planning, giving all tasks the time to accomplish, and developing cues for transition are all good clinical tips towards solving the challenges. Additionally, encouraging the youth to elaborate on conversations with detail to improve language and communication skills can be useful particularly in withstanding and recounting negative experiences.

\section{B.I. Mental flexibility and feedback circuitry}

Mental flexibility is a specific sub-domain of executive function that represents the ability to shift from one task to another on demand, without being rigid and inflexible or yield to frustration due to feedback or any other emotional interference. Patients with BD showed increased DLPFCACC-parietal activity, recruiting regions responsible for cognitive control and visuospatial strategies [35]. This maladaptive activity is greater in response to failed reversal trials paired with punishment, i.e., losing points, illustrated with increased activity in precuneus and posterior cingulate cortex. There may be no difference in $\mathrm{BD}$ and severe mood dysregulation (SMD) in the level of decreased caudate activity considered responsible for switching and learning new rules.

\section{B.II. Clinical translation based on brain function}

Both the anterior cognitive control regions and posterior visuospatial and attentional hubs are involved in expending cognitive effort in individuals affected with BD and show clear dysfunction in subcortical regions responsible for adaptation. The ability to switch when required is a problem in severe affect regulation, be it SMD or BD. These findings offer a sketch line of where "the engine is at fault" in $\mathrm{BD}$.

\section{Attention}

\section{A.I. Behavioral findings}

Two aspects of attention were shown to be impaired in BD.

The first attentional impairment is the inability to sustain attention that includes poor vigilance, false responding with target insensitivity, 


\section{A.II. Clinical translation based on behavioral findings}

perseverance, and variable intraindividual response timing [12-14, 25, 36]. The second attentional impairment is the selective attention or bias to negative stimuli such as angry faces [37], negative words [38], problem solving when exposed to negative words [7], or matching emotional words [39]. Attentional bias to angry faces was shown in BD with anxiety, though not among those with BD without anxiety [40]. Additionally, bias away from positive self-referential words has been reported in $\mathrm{BD}$ with depressed symptoms [38]. Selective attentional bias has, therefore, been demonstrated across domains of executive function and in the context of internalizing symptoms of depression and anxiety. Selective bias seems especially impaired when the target is emotionally valanced and worse if the emotion is negative [39], but not when the target is emotionally neutral [29].

Lower persistence or maintenance of focus and being easily swayed by environmental "in the face" negative stimuli in interpersonal interactions can be a hindrance to optimal functioning. Additionally, comorbid internalizing emotional states may also lead to selective bias, based on internal state of depression or anxiety.

\section{Impulse control}

\section{A.I. Behavioral findings}

4. A.II. Clinical translation based on behavioral findings

This is a problem both in the form of emotionally explosive verbal expressions and physical outbursts with poor self-control. Impulse control has been examined with various versions of Stop-Signal Task that tests for the ability to inhibit a motor act that is already on the way to be executed. In $\mathrm{BD}$, findings consistently indicate decreased accuracy and increased intrasubject variability in response times. Poor attention to performance monitoring and inability to delay gratification may also contribute to premature reactivity or impulsivity. Impulsivity, while common in both the ADHD and $\mathrm{BD}$, may arise from differential engagement of affective and cognitive systems in these disorders [1•].

\section{A.tl. Clinical translation based on behavioral findings}

While the clinical features of impulsivity per se were not correlated with the response inhibition measures, behavioral findings indicated poor inhibition in stopping the action midway when cues were given to stop. Behaviorally, when patients with BD get going, they cannot stop, and potentially, this pattern correlates with disinhibition and inattention. These elements are transferred to treatment strategies in exercising cognitive control with awareness in decision-making and measuring consequences.

3 and 4. B.I. Attention-impulse control-response inhibition circuitry

Regardless of the task probing response inhibition or the type of sample within bipolar spectrum disorders in youth, there is consistency in the results that indicate VLPFC and ACC dysfunction. The dual role of emotional and motor control of VLPFC region and the dual role of emotional 
3 and 4. B.II. Clinical translation based on brain function

processing and cognitive error correction by ACC may explain the involvement of these two regions in modulating both emotions and response inhibition.

Two contiguous pathophysiological processes implicate PFC regions in cognitive control and temporal-parietal regions in scanning with attention. Relative to those with ADHD who demonstrate cognitively driven impulsivity, people with BD suffer from both cognitive and emotional impulsivity, thereby engaging fronto-striatal [41-43] and fronto-limbic [44-46] circuitry regions.

Inattention and failure to inhibit impulsive responses are common deficits either due to comorbid ADHD or specific cognitive deficits that are prominent in early-onset $\mathrm{BD}[2,5,14,47]$.

Given that $\mathrm{BD}$ is an emotional and cognitive illness, regions that have dual roles in both the emotional and cognitive processes such as higher cortical centers like VLPFC, intermediary ACC, and the subcortical regions such as striatum are all impaired in the illness. They were shown to increase in activity with effort or decrease in activity if the patients fail to inhibit. When attention is deployed, higher frontal cortical regions such as VLPFC and DLPFC appear to be under-functioning and posterior cortical regions such as temporal and parietal regions and/or subcortical region such as basal ganglia may be deployed to compensate. Treatment interventions may serve well to target mood regulation, cognitive control, and error detection to improve the brain function.

\section{Working memory}

\section{A.I. Behavioral findings}

\section{A.II. Clinical translation based on behavioral findings}

Working memory or the ability to hold information on line for short periods of time while accomplishing the task are critical for children's learning and day-to-day operations. We were able to show that verbal working memory was associated with poor reading and reduced vigilance with math difficulties [25]. Working memory did not catch up with healthy individuals with standard treatment in patients with $\mathrm{BD}$, and in fact, verbal memory remained worse on 3-year follow-up [14, 25].

Educational achievement is dependent on the "hard wired" difficulties that the student brings to the class, and the literature informs us that both reading and math are impacted by the ability to hold information in the multistepped tasks required for learning. Besides, angry facial expressions lower the ability to potentially regulate emotions that impact working memory.

\section{B.I. Working memory circuitry}

Working memory deficits in BD are linked to impaired DLPFC-ACCthalamic-basal ganglia circuitry [23, 48]. In BD, relative to ADHD, tasks involving working memory under emotional challenge engaged the emotional circuitry regions within the ventral PFC and subgenual cingulate [49]. 


\section{B.II. Clinical translation based on brain function}

In contrast, in ADHD, these tasks engaged only the dorsal cognitive frontostriatal and frontoparietal circuits. Interestingly, when BD or ADHD is compared to healthy individuals, both the cognitive and emotional regions are underactive.

Also, in $\mathrm{BD}$, the cognitive and affective regions that are underactive with angry/negative emotions are overactive with happy emotions. It is a consistent finding in $\mathrm{BD}$ that negative stimuli have greater impact on the prefrontal regions and may "switch off" from activation or functioning while positive stimuli engage the PFC regions with increased activity.

A study unraveling the brain network connectivity showed that the affective network circuitry (VLPFC-amygdala) and the facial emotion processing circuitry (fronto-temporo-amygdala) regions are not "firing" in synchrony with the rest of the corresponding brain network regions in $\mathrm{BD}$ during the working memory task [49]. Simultaneously, working memory circuitry is over-engaged or hyperconnected [50]. This pattern of abnormalities illustrates interlinked compensatory processes at the interface of emotional and cognitive networks in $\mathrm{BD}$.

The brain functional studies show that working memory and affective circuitries are both involved in $\mathrm{BD}$ and $\mathrm{ADHD}$, though affective circuits are malfunctioning to a greater degree in $\mathrm{BD}$. Negative stimuli impair the ability of PFC regions to function effectively in BD. Working memory tasks take a toll on affective circuitry synchrony especially when the patients are biased by negative environmental stimuli. Reducing working memory exertion and negative environmental stimuli may improve brain function in $\mathrm{BD}$. The findings also explain how ADHD shares overlapping pathophysiology with $\mathrm{BD}$, a finding that is valuable in educating the parents on the features of $\mathrm{BD}$ with or without ADHD.

\section{Reward and risk response}

\section{A.I. Behavioral findings}

Several studies have illustrated how patients with BD fail to grasp reinforcement expectancy and shift away from the correct responses, regardless of whether they were related to reward or unexpected loss. Additionally, feedback sensitivity dropped significantly if there was frustration induced by rigged feedback $[19 \bullet \bullet, 29]$ or if patients were distracted by taskincongruent stimuli [30].

\section{A.II. Clinical translation based on behavioral findings}

While experimental studies focus on understanding responses to reward and punishment, their findings are relevant in managing BD patients. These observations from the behavioral and fMRI studies have radically changed the way we conceptualize reward and punishment in individuals with affect dysregulation. Children with $\mathrm{BD}$ are impaired in their ability to tolerate negative stimuli, to exert inhibitory control, and to shift their attentional and behavioral focus so as to adapt to changes, while they are motivated by immediate reward or gratification. Solution to behavioral modulation with 
6. B.I. Reward processing and positive valence circuitry

reward and risk can be developed through careful understanding of multidomain dysfunction beyond the over reactivity to risk or negative consequences and inability to learn and predict outcome based on reward or risk.

Reward processing involves several components such as emotional evaluation, motivation, and decision-making. In case of pediatric BD, dysfunction could be at the interface of the reward-centric ventromedial PFC (VMPFC or orbitofrontal cortex, OFC)-ventral striatal circuitry and the emotion-centric DLPFC-VLPFC-ACC-limbic circuitry. In addition to the studies in pediatric $\mathrm{BD}$, normative studies illustrate greater activation in the VMPFC, the amygdala, and nucleus accumbens during winning than losing. Both in adults and adolescents, the degree of activation is proportional to reward [51, 52]. However, striatal regions show reduced activation during reward anticipation in adolescents relative to adults [52]. The BD patients may show altered evaluation and appraisal of reward contingencies with impaired consummation resulting in excessive excitability or frustration and impaired motivation illustrated in response to anticipation, possibly due to poor executive function.

\section{B.II. Clinical translation based on brain function}

Delineation of the underlying motivational and consequence-based physiological regulatory processes is critical to understanding how behavior is shaped in response to any given environment. Emotional reactivity with extreme highs and lows, associated hyper-arousal, and ill-timed responses to altered mental appraisal are potentially relevant and can be explained by deficits in fronto-temporo-parieto-subcortical circuits. The ventral circuits, i.e., fronto-limbic and fronto-striatal circuits, may be predominantly involved in BD [44]. The increased incidence of substance abuse in pediatric bipolar disorder (PBD), and the increased intensity in seeking reward (often described by parents as "mission mode" or "obsession"), or explosive behavior if reward is denied can be explained by the complex brain pathophysiology underlying the anticipation and reward or loss. The link between the behavioral abnormalities with the impairment in reward circuitry requires further study. However, current evidence paired with clinical understanding of $\mathrm{BD}$ patients generates novel hypotheses. To give an example, a child with BD may repeatedly insist that his parents should purchase a video game and such incessant requests are often referred to as "obsessions" by parents. This behavior can be considered as arising from an inability to postpone gratification paired with poor inhibition control (VLPFC-straital impairment). If the reward is not obtained, patients may show excessive reactivity presumably because of the impaired affective circuitry (VLPFC-amygdala impairment).

7. Perspective taking

Perspective taking is the ability to understand the point of view of others, which is a dimension of "Theory of Mind" [53, 54]. While there could be 
7. A.II. Clinical translation based on behavioral findings

some fine differences in some domains, the fundamental dysfunction in the affective systems may be similar among individuals with severe affect dysregulation and individuals with $\mathrm{BD}$ due to the shared involvement of amygdala, VLPFC, and emotion processing circuits. In fact, subjects with severe affect dysregulation show deficits across social reciprocity tasks involving social awareness, social cognition, social communication, and social motivation ([55] Whitney et al. 2013). We showed that the real intentions behind subtle hints especially during negatively valenced affective story task were poorly recognized by BD patients [53]. A subsequent study showed clear link between poor performance on the Cognitive and Emotional Perspective Taking Task and the Index of Peer Relations in BD type I relative to healthy controls, but not so in BD type II [54]. Irrespective of the emotional valence, $\mathrm{BD}$ type I had poor ability to understand the mental state of others, which is not the case in BD type II.

These theory of mind deficits suggest that BD may also need to be differentiated from autism spectrum disorders, especially as abnormal social perspective taking combined with rigidity is part of autism "spectrum." Both the aforementioned rigidity and poor executive functioning coupled with decreased ability to grasp the social nuances and act with reciprocal understanding, and affect regulation when challenged, can complicate the clinical picture.

\section{Interface of emotional and cognitive operations}

\section{A.I. Behavioral findings}

\section{A.II. Clinical translation based on behavioral findings}

The cognitive-affective interface is especially well examined in the fMRI studies summarized below and equally highlighted through the studies on affective disturbance in ADHD [1•]. In BD, cognitive task probes seem to engage not only the cognitive but also the affective systems. Additionally, several studies in the past decade have examined domains of cognition under emotional challenge with consistent and robust findings [14, 19••, 25]. Negative faces deterred working memory in both BD types I and II [56]. Negative feedback of failure or loss triggered worsening in attention.

In case of $\mathrm{BD}$, the struggle to focus, keep things on line, problem solve, and manage distractions is constantly challenged by emotional dysregulation, especially if untreated. These children are highly vulnerable to negative environmental cues and require help to manage their mood, accept criticism, and mindfully focus on the cognitive tasks at hand.

\section{B.I. Interface of emotion and cognitive circuitry}

The experiments described previously involving attentional control and problem solving under negative emotional challenge illustrate the joint dysfunction of cognitive and emotional circuitry in pediatric $\mathrm{BD}$. When $\mathrm{BD}$ and healthy subjects are compared directly, the pregenual ACC is overactive while limbic overactivity is significant mainly with negative and not with 
8. B.II. Clinical translation based on brain function

positive emotions. These results suggest that in $\mathrm{BD}$, negative emotions may influence cognitive systems more than positive emotions. Especially, VLPFC dysfunction distinguishes BD from ADHD with over- and underactivity, very similar to that seen when probing working memory $[49,57]$.

These results are informative for illustrating how negative emotional stimuli can destabilize cognitive ability in youth with BD. In fact, both BD and ADHD groups show higher cortical dysfunction with either positive or negative emotions, with abnormal VLPFC activation being specifically implicated in BD. These results suggest, indirectly, several ideas that can be potentially transferred to real-life settings: (1) negative consequences may result in poor functioning of cortical cognitive control regions along with emotion control regions of the brain. This dysfunction could be originating from abnormalities either in the amygdala or the PFC or both. (2) Psychotherapy must be crafted with the principles of building on strengths, using the right timing, tone of voice, and measured language with any corrective feedback. It is a major paradigm shift in removing immediate negative consequences; (3) a compassionate teaching style may be more conducive to learning than imposing a strict structure that may engender conflict and negative emotional exchanges. These are hypotheses generating suggestions that aim to steer research towards solving real-life problems.

\section{Chemotherapy or pharmacotherapy}

\section{Medication for affect regulation: neuroplasticity}

The effect of medications on brain function suggests that neuroplastic changes are associated with clinical recovery $[58,59]$. For example, affect regulation probed during a response/impulse control task showed that lamotrigine increased activity in the medial prefrontal cortex (MPFC) and pregenual, subgenual, and posterior cingulate cortex activity (PCC) over 14 weeks in BD mania, while striatal activity was increased in healthy controls [60]. In the case of bipolar depression, MPFC, DLPFC, striatum, and PCC showed increased activation from baseline [61]. These results show a particular promise that lamotrigine may be useful in those suffering from mania and later develop depression probably because of its special ability to normalize activity in the MPFC, the area crucial to regulating amygdala activity and in reducing ruminations.

In another double-blind randomized trial, divalproex sodium (DVPX) showed increased connectivity of the subgenual cortex within the affective evaluation and inhibition circuitry during response inhibition [62]. This is similar to the earlier outcome with lamotrigine, confirming that antiepileptic agents may influence subgenual cortical function during impulse control in BD. The antipsychotic risperidone is associated with increased engagement of insula (relative to DVPX group) during response inhibition. Arousal associated with aggression in mania predictably responds to risperidone, and these results are reassuring as they align with clinical experience.

At the interface of executive function and affective processing domains, evaluative MPFC and ACC were engaged during negative word matching with all drugs, 
i.e., risperidone, DVPX, and lamotrigine $[44,63]$. However, under the negative emotional challenge, cognitive DLPFC activation response was reduced with lamotrigine and risperidone. This suggests that cognitive enhancers may be needed post-mood stabilization. This paves the way for the use of stimulants after mood stabilization through a problem-solving algorithm.

Furthermore, prognostic markers of clinical outcome are beginning to emerge, with greater amygdala activity at baseline being a marker for clinical response to risperidone possibly involving greater effort in reducing amygdala hyperactivation. Greater MPFC activity at baseline was a marker for a good outcome with DVPX where preserved higher cortical region could potentially modulate affective system more effectively. With all medications, the amygdala showed reduced activity from baseline along with recovery from manic state, but remained active relative to that of healthy individuals (trait marker), while the activation of PFC regions normalized with mood stabilization (state marker) [22•]. However, long-term treatment for at least 4 months with a standardized algorithm led to increased amygdala connectivity [64] in the affective circuitry and normalized subcortical activity [65]. These results indicate the need to maintain mood stabilization for longer periods of time, i.e., 4 months to 3 years.

A problem-solving forum is provided based on empirical research of individual studies as well as empirically based pharmacotherapy algorithm [1・], updated based on emerging literature. The primary goal is to target emotion dysregulation and stabilize the commonly occurring rapid cycling mood in manic or mixed episodes. Even prior to that, prescription hygiene is critical such as stopping antidepressants or stimulants that may worsen mood regulation $[66,67]$.

1. Second-generation antipsychotics (SGAs): The first choice for affect instability in PBD is SGAs as postulated by the National Institute for Health and Care Excellence (NICE) guidelines [68]. It is now established that SGAs offer the advantage of rapid onset of action, with proven efficacy. Metabolic side effects such as increased appetite, weight gain and increased cholesterol, extrapyramidal side effects, and sedation can all be the adverse effects of SGA, though tolerability may vary across different individuals and different antipsychotic agents (see Table 1). At the neurochemical level, most SGAs are serotonin-dopamine antagonists. D2 receptor blockade in the mesolimbic pathway is thought to be important for reducing manic symptoms, while the serotonin $2 \mathrm{~A}$ antagonism leads to indirect increase of dopamine in the nigrostriatal pathway, resulting in fewer extrapyramidal side effects compared to firstgeneration antipsychotics.

Role of antiepileptic medications: Severe symptomatology is addressed with combination treatment of an antiepileptic (oxcarbazepine, lamotrigine, carbamazepine, divalproex sodium) or lithium along with the SGA, as first choice. Tolerance may be induced by chronic high doses due to post receptor sensitivity [69]. Additionally, SGAs can serve as mood stabilizers in addressing bipolar depression, prolong the relapse 


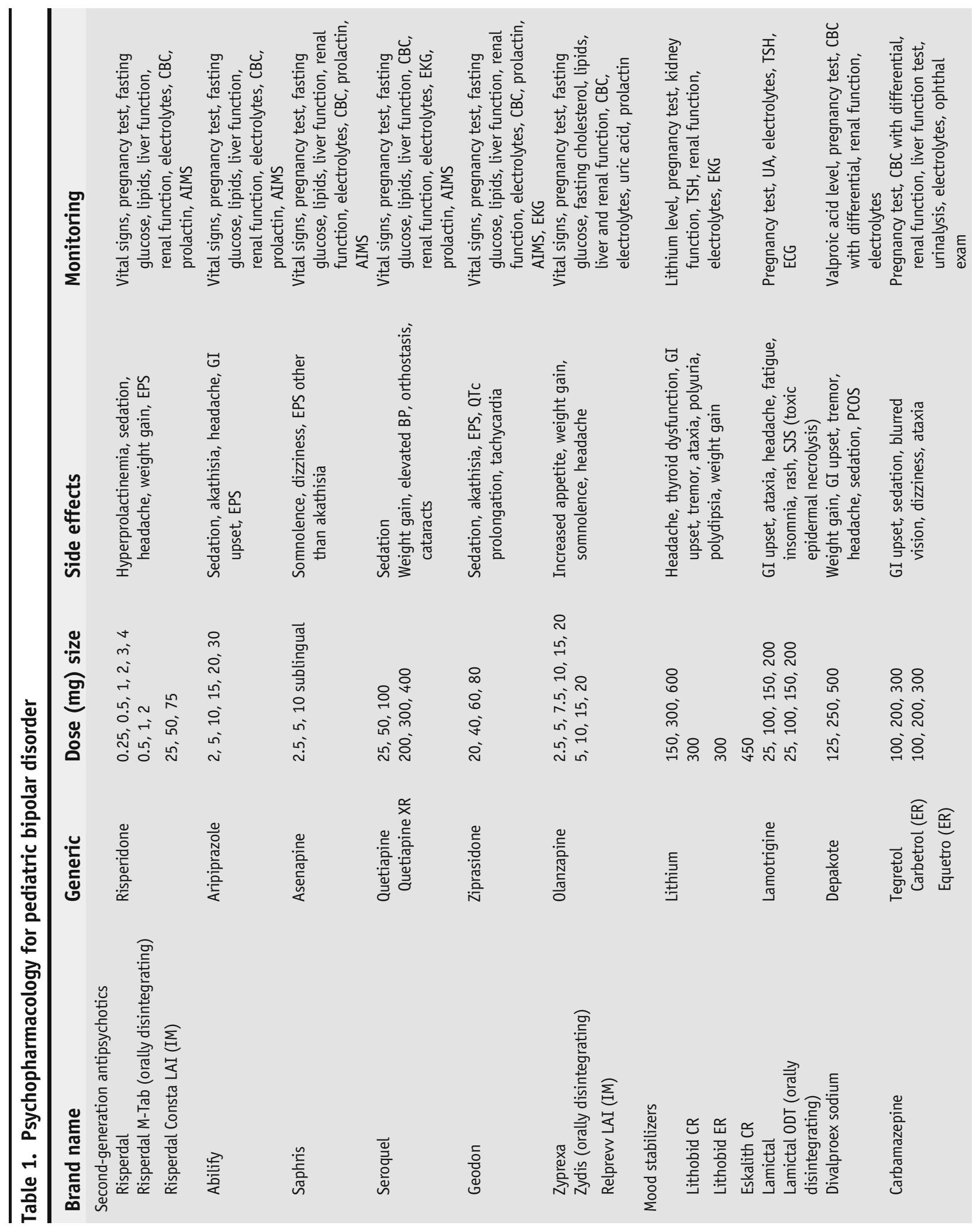




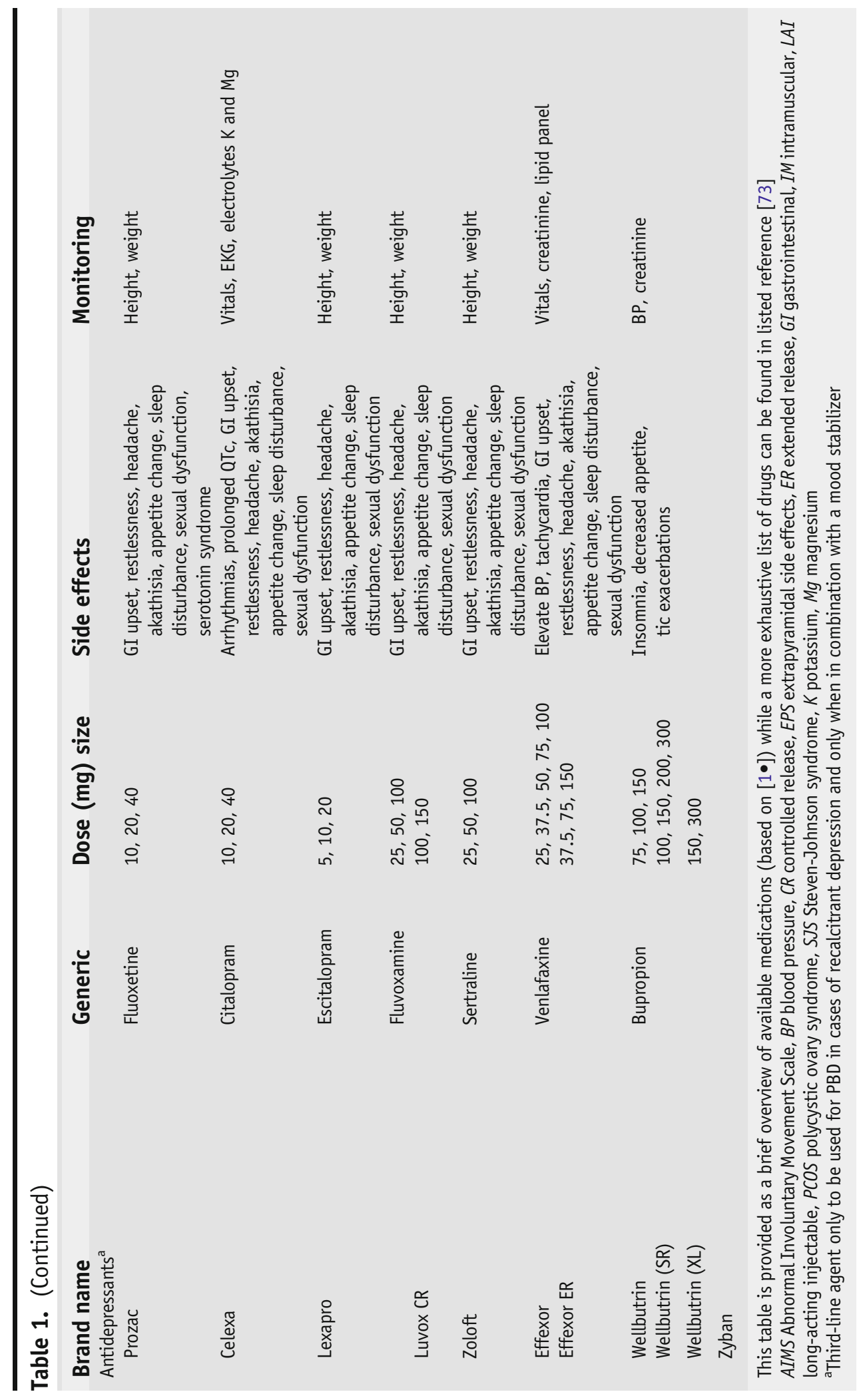


time, and reduce suicidal behavior [70, 71]. During the maintenance phase, patients may be stabilized on monotherapy with either an antiepileptic, lithium, or SGA. With regard to mechanism of action, lithium has been shown to affect several interlinked signaling cascades, such as phosphoinositide and adenylyl cyclase. Furthermore, lithium response may be influenced by polymorphisms of the inositol poly-phosphate 1phosphatase gene located on chromosome $2 \mathrm{q} 32$; CREB1 gene on $2 \mathrm{q} 32$ 34 ; glycogen synthase kinase (GSK)-3 $\beta$ gene on $3 \mathrm{q} 13,7 \mathrm{q} 11.2$, and 3 25, and val66Met of brain-derived nerve growth factor (BDNF) gene $[72,73]$. Lack of response to lithium may be more likely to occur in subjects with the s allele of 5-HTTLPR with val/val genotype of BDNF. Most antiepileptic agents act through calcium channel blockade and are thought to improve electrochemical balance at the cellular level.

2. Treating problems associated with affect dysregulation: Hyperarousal, aggressive outbursts, and explosive behavior usually necessitate optimization of the primary mood-stabilizing medication. Adding alpha agonists such as clonidine, guanfacine, or other long-acting variants (e.g., Kapvay ${ }^{\mathrm{Tu}}$ or Intuniv $^{\mathrm{TH}}$ ) may further reduce reactivity and arousal. These medications are typically administered in facilitating transition from home to school with morning dose, from school to home with evening dose, or late night dose for sleep initiation. While there are no standardized guidelines for use in youth, lower doses are preferred. These medications aid in reducing the need to increase the dose of SGA or mood stabilizers by increasing dopamine in the prefrontal cortex [74]. They are often used as adjuvants and alongside stimulant medications to help improve attention.

3. Treating depression: Depressive symptoms in BD may be present during a mixed episodes or during major depressive episodes (MDE). The residual symptoms of mania or comorbid ADHD masking MDE in pediatric BD may be difficult to disentangle. Lamotrigine and lithium are considered first-line mood stabilizers for the treatment of bipolar depression. In pediatric BD presenting with recalcitrant MDE and comorbid anxiety disorders, a small dose of selective serotonin reuptake inhibitors (SSRIs) may be prescribed subsequent to mood stabilization. Compared to fluoxetine, sertraline, and fluvoxamine, citalopram and long-acting escitalopram are more selective potentially causing less activation and have less drug interactions as they are metabolized by CYP 2C9 and C19 and not CYP 2D6 that metabolizes SGAs [74]. A recent double-blind placebo-controlled trial of olanzapine/fluoxetine combination showed statistically significant reduction in depression relative to placebo in 1017 -year-old youth with bipolar depression [75••]. The most frequent treatment-emergent adverse events with the olanzapine/fluoxetine combination were weight gain, increased appetite, and somnolence.

\section{Inattention, impulse control, and hyperactivity: a symptomatic treatment of comorbid ADHD}

1. Psychostimulants: Currently, the medications of choice for ADHD are long-acting stimulant preparations taken as a single dose in the morning. 
This dosing schedule increases adherence, reduces interference with other class activities, and avoids the embarrassment of going to the nurse's office. The second dose is administered as an immediate release preparation in lower dose, around $4 \mathrm{pm}$ to accomplish homework. Long-acting or short-acting preparations are four groups of compounds: D-isomer of methylphenidate (Focalin ${ }^{\mathrm{TH}}$ ), methylphenidate (Ritalin ${ }^{\mathrm{TM}}$, Daytrana $^{\mathrm{TM}}$, Metadate $^{\mathrm{TM}}$, Concerta ${ }^{\mathrm{TM}}$, Quillivant $\left.\mathrm{XR}^{\mathrm{TM}}\right)$, mixed amphetamine salts (dextroamphetamine and amphetamine, Adderall ${ }^{\mathrm{TM}}$;

lisdexamfetamine dimesylate, Vyvanse ${ }^{\mathrm{TM}}$ ), and dextroamphetamine (Dexedrine $^{\mathrm{TH}}$ ). The chemical compounds and various delivery mechanisms (i.e., long-acting tablets, capsules, sprinkles, or patches) impact each individual differently. No comprehensive data set exists to empirically suggest one compound versus another. Of note, there are important differences in dosing: mixed amphetamine salts dosed at 0.1 to $0.2 \mathrm{mg} / \mathrm{kg}$ body weight, methylphenidate dosed at 0.3 to $1 \mathrm{mg} / \mathrm{kg}$ body weight, and dextroamphetamine dosed at 0.15 to $1.5 \mathrm{mg} / \mathrm{kg}$ body weight. Lower doses help increase attention while higher doses improve social behavior.

Although the known side effects are similar across the compounds, tolerability varies across individuals. Common side effects include activation, difficulty sleeping, and decreased appetite. Decreased appetite can be addressed by eating prior to taking the medication or after the medication has worn off (generally less than 12-h half-life). High doses precipitate affect dysregulation which may mimic hyperactivity. Continuing to increase the stimulant dose may lead to further clinical deterioration. Stimulants may aggravate irritability either immediately or over several weeks of treatment.

2. Non-stimulant medications: Agents that modulate dopaminergicnoradrenergic transmission such as bupropion or mainly the noradrenergic transmission such as atomoxetine could be options for comorbid ADHD. A therapeutic advantage of these medications is the lack of abuse potential. The onset of action is slower than stimulants, typically $2-$ 4 weeks. Atomoxetine is a potent inhibitor of the pre-synaptic norepinephrine transporters in the brain with minimal affinity for other neurotransporters and neuroreceptors [74]. Bupropion is a weak norepinephrine-dopamine reuptake inhibitor (NDRI) and may also induce the release of dopamine and norepinephrine.

The following tests are completed at the initial assessment and every 3 months. SGA monitoring requires a complete blood count, liver function tests, lipid panel, and renal function tests. Prolactin is commonly elevated with risperidone compared to the other SGAs, and it is advisable to test prolactin levels every 3 months while patients are in treatment with this agent. Generally, however, it is a good practice to obtain baseline and follow-up levels of prolactin during the first 6 months and yearly afterwards for all SGAs. Lithium and valproic acid levels should be ordered if 
prescribed. For lithium, blood levels are used to ensure the prescribed doses remain within the therapeutic window and avoid acute toxicity. Additionally, urine creatinine levels are also required every 3 months due to the potentially nephrotoxic effect of lithium. Routine urine drug screens and urine pregnancy tests (for females, post-menarche) are also recommended as the information is relevant to pharmacotherapy choices. For older youth, non-prescribed stimulant abuse and abuse of other illicit drugs should be considered in the differential diagnosis. It is suggested that an EKG is requested if prescribing ziprasidone and for all patients with personal or family history of cardiac problems.

\section{Delivery and dynamics of treatment}

A realistic approach to clinical practice embraces the fact that adherence to protocol-based treatments must be paired with thoughtful understanding of the dynamics of the family's relationships and stressors. The index illness of pediatric BD is a stressor in itself and may precipitate further relational stresses such as parental divorce. Assessing the subtleties of the family's ecosystem and offering validation, support and practical suggestions can be the key to a successful individualized intervention. An unspoken element of treatment is the method of delivery with three universal components for a good outcome: (1) a compassionate, non-judgmental, and careful analysis to uncover interpersonal dynamics; (2) balance of gentle yet forthright feedback paired with thoughtful insight that would prompt change from status quo; and (3) genuine assessment of family strengths, offering insight to the family so that they can find these elements of strength and then use them to repair or work around entrenched maladaptive patterns.

Neuroscience informed empirical treatment for pediatric $B D$

In youth with $\mathrm{BD}$, mood regulation is the key objective. Child- and familyfocused cognitive behavioral therapy (CBT) (CFF-CBT) offers a model that centers on parental validation and encourages them to become the coach for their child by integrating psychoeducation, CBT, interpersonal therapy, mindfulness, family therapy, and positive psychology. Cognitive restructuring is used to problem-solve. The most powerful and potentially most challenging component of the self-psychology/CBT model is the "no negative consequences" approach. As the behavior of youth with $\mathrm{BD}$ can be challenging, it is easy to focus on these components and to respond by negative criticism or punishments. Punishment only further exacerbates emotional reactivity and arousal in the affected youth. Instead, an approach fostering rational thinking with an emotional buffer of interpersonal support is preferable. Modeling these healthy approaches with families will help them to mirror positive interactions and form new patterns of behavior. The CFF-CBT involves education about the neurobiological understanding of how emotions cloud the thinking (VLPFC and DLPFC interface and fronto-limbic dysfunction as described above) and how affect dysregulation and poor decision-making co-occur (interlinked dysfunction of affective and cognitive domains). The RAINBOW therapy is a mnemonic 
for the components of CFF-CBT therapy sessions to both parents and children. The components of RAINBOW therapy are the following: $\mathrm{R}=$ the importance of routine (includes sleep hygiene), $\mathrm{A}=$ affect regulation/anger control (includes knowledge about the disorder, medication, life charts), I="I can do it" (positive self-statements and self-stories), $\mathrm{N}=$ no negative thoughts (restructuring negative thinking/living in the "now"), $\mathrm{B}=\mathrm{be}$ a good friend (balanced lifestyle: for parents), $\mathrm{O}=$ "Oh, how can we solve it?" (interpersonal and situational problem solving), and W=ways to ask and get support (building a support network).

The reliability of the RAINBOW program has been demonstrated during both a short- and long-term efficacy trial. A short-term open trial of RAINBOW treatment for 12 weeks in a group setting resulted in a reduction in mania scores [76]. Global functioning scores across domains of mania, depression, ADHD, psychosis, aggression, and sleep disturbances significantly decreased following the initial 12 weeks and were subsequently sustained for 3 years [77]. The longevity of the positive effects following the initial trial suggests feasibility of a maintenance model for RAINBOW.

Another option is Dialectic Behavior Therapy (DBT) along with medication management. For pediatric BD patients, this treatment strategy resulted in significantly less severe depressive symptoms on follow-up and nearly three times less likely to demonstrate suicidal ideation [78•]. Over the follow-up period, evidence indicates a large effect size in prolonging periods in a euthymic state. The combination of family psychoeducation and skill building yielded one of the most efficacious modalities (i.e., Multi-Family Psychoeducational Psychotherapy, Family-Focused Treatment, CBT with interpersonal therapy) for pediatric BD patients [79•]). In terms of future directions, interpersonal and social rhythm therapy (IPSRT) is being further explored and tested.

In $\mathrm{BD}$, especially in youth, it is a rule than exception that along with the symptoms of the $\mathrm{BD}$, multiple brain domains are affected that impair daily function. What is not addressed by the aforementioned protocols is the invariable complexity encumbered by multi-domain involvement at an individual level.

To address the emotion dysregulation, empirical findings led to the development of RAINBOW therapy and problem-solving pharmacotherapy for addressing symptoms of $\mathrm{BD}$ and potential comorbidities. Just as not all symptoms need to be present to diagnose $\mathrm{BD}$, not all domains may be affected at any individual level. To tackle the cognitive problems, tutoring for multi-stepped learning, reducing homework to decrease cognitive burden and increasing motivation through success, increasing test taking duration, helping with problem solving through preparation and planning, giving all tasks the time to accomplish, and developing cues for transition are all good clinical strategies. Considering the critical dysfunction at the interface of emotional and cognitive systems, there is abundant evidence that negative emotions impair cognitive function. This demands not only the abovementioned strategies but also environmental manipulation where parents could choose the right fit of teaching 
methods and personnel where possible. An array of options summarized above and tailored to the individual patient and their unique system of caretakers is required to arrive at a personalized approach. Accurate assessment (A), educating the families in a simplified language on the complex neurobiology such as brain and behavioral dysfunction (B) that underlies emotional and cognitive problems, and chemical intervention (C) paired with dynamic psychosocial interventions (D) will lead to precision in addressing the individual and family's needs. Recognizing that impulse control is closely linked to reward seeking, working memory is linked to sustain attention and execute the actions, and face emotion processing, perspective taking, and emotion regulation operate conjointly reflecting the complexity of brain operations and environmental impact. While no domain functions in isolation, ultimately, it is the understanding of all the elements that help recognize the complexity of interlinked domain function of the BD as well as the environmental impact at an individual level towards precision care in research or clinical application.

\section{Compliance with Ethics Guidelines}

\section{Conflict of Interest}

Dr. Pavuluri receives or has received grant support from the following sources: National Institutes of Health (NIH), the National Institute of Mental Health, National Alliance for Research on Schizophrenia and Depression (NARSAD)/Brain and Behavior Research Foundation, American Foundation for Suicide Prevention, and Marshall Reynolds Foundation. Due to multiple invitations to contribute on related topics, there could be overlapping concepts/text that are appropriately cited to reflect the source.

\section{Human and Animal Rights and Informed Consent}

This article does not contain any studies with animal subjects performed by the author.

\section{References and Recommended Reading}

Papers of particular interest, published recently, have been highlighted as:

- Of importance

• Of major importance

1. Pavuluri MN, May A. Differential treatment of bipolar disorder and attention deficit hyperactivity disorder. Psychiatr Ann. 44 (10):471-480.8.

Offers an overview of neurobiology of bipolar disorder and attention deficit hyperactivity disorder with pragmatic steps based on empirical data.

2. Birmaher B. Bipolar disorder in children and adolescents. Child Adolesc Mental Health. 2013;18(3).

3.• Sala R, Axelson D, Birmaher B. Phenomenology, longitudinal course, and outcome of children and adolescents with bipolar spectrum disorders. Child Adolesc Psychiatr Clin N Am. 2009;18(2):273-89.
A classic article on the trajectory of complex bipolar disorder.

4. Birmaher B, Axelson D, Goldstein B, et al. Four-year longitudinal course of children and adolescents with bipolar spectrum disorders: the Course and Outcome of Bipolar Youth (COBY) study. Am J Psychiatry. 2009; 166(7):795-804.

5. Geller B, Tillman R, Bolhofner K, Zimerman B. Child bipolar I disorder: prospective continuity with adult bipolar I disorder; characteristics of second and third episodes; predictors of 8-year outcome. Arch Gen Psychiatry. 2008;65(10):1125-33. 
6. American Psychiatric Association. Diagnostic and statistical manual of mental disorders. 5th ed. Arlington: American Psychiatric Publishing; 2013.

7. Pavuluri MN, Oconnor-Marlow M, Harral E, Sweeney JA. An fMRI study of the interface between affective and cognitive neural circuitry in pediatric bipolar disorder Psychiatric Res Neuroimaging. 2008;162(3):244-55.

8. West AE, Schenkel LS, Pavuluri MN. Early childhood temperament in pediatric bipolar disorder and attention deficit hyperactivity disorder. J Clin Psychol. 2008;64:402-21.

9. Lochman JE, Evans SC, Burke JD, Roberts MC, Fite PJ, Reed GM, et al. An empirically based alternative to DSM-5's disruptive mood dysregulation disorder for ICD-11. World Psychiatry. 2015;14(1):30-3.

10. Stifter CA, Dollar JM, Cipriano EA. Temperament and emotion regulation: the role of autonomic nervous system reactivity. Dev Psychobiol. 2011;53(3):266-79.

11. Findling RL, Gracious BL, McNamara NK, Youngstrom EA, Demeter CA, Branicky LA, et al. Rapid, continuous cycling and psychiatric co-morbidity in pediatric bipolar I disorder. Bipolar Disord. 2001;3(4):202-10.

12. Dickstein DP, Treland JE, Snow J, McClure EB, Mehta MS, Towbin KE, et al. Neuropsychological performance in pediatric bipolar disorder. Biol Psychiatry.

2004;55(1):32-9.

13. Doyle AE, Wilens TE, Kwon A, Seidman LJ, Faraone SV Fried R, et al. Neuropsychological functioning in youth with bipolar disorder. Biol Psychiatry.

2005;58(7):540-8.

14. Pavuluri MN, West A, Hill K, Jindal K, Sweeney JA. Neurocognitive function in pediatric bipolar disorder: three-year follow-up shows cognitive development lagging behind healthy youth. J Am Acad Child Adolesc Psychiatry. 2009;48(3):299-307.

15. Smith DJ, Muir WJ, Blackwood DH. Neurocognitive impairment in euthymic young adults with bipolar spectrum disorder and recurrent major depressive disorder. Bipolar Disord. 2006;8(1):40-6.

16. Geller B, Warner K, Williams M, Zimerman B. Prepubertal and young adolescent bipolarity versus ADHD: assessment and validity using the WASH-U-KSADS, CBCL and TRF. J Affect Disord. 1998;51(2):93-100.

17. Pavuluri MN, O'Connor MM, Harral EM, Moss M, Sweeney JA. Impact of neurocognitive function on academic difficulties in pediatric bipolar disorder: a clinical translation. Biol Psychiatry. 2006;60(9):951-6.

18. McClure EB, Treland JE, Snow J, Schmajuk M, Dickstein DP, Towbin KE, et al. Deficits in social cognition and response flexibility in pediatric bipolar disorder. Am J Psychiatry. 2005;162(9):1644-51.

19.• Rich BA, Schmajuk M, Perez-Edgar KE, Pine DS, Fox NA, Leibenluft E. The impact of reward, punishment, and frustration on attention in pediatric bipolar disorder. Biol Psychiatry. 2005;58(7):532-9.

An influential article on the impact of emotion on cognition

20. Schenkel LS, Pavuluri MN, Herbener ES, Harral EM, Sweeney JA. Facial emotion processing in acutely ill and euthymic patients with pediatric bipolar disorder. J
Am Acad Child Adolesc Psychiatry.

2007;46(8):1070-9.

21. Schenkel LS, West AE, Jacobs R, Sweeney JA, Pavuluri $\mathrm{MN}$. Cognitive dysfunction is worse among pediatric patients with bipolar disorder type I than type II. J Child Psychol Psychiatry. 2012;53(7):775-81.

22. Pavuluri MN. Chapter 13: Neurobiology of bipolar disorder in youth. In: Strakowski S, Adler C, Del Bello $\mathrm{M}$, editors. Bipolar disorder in youth, 1st edition. NYC: Oxford Press; 2014.

A one place review that offers update on the neurobiology of pediatric bipolar disorder.

23. Chang K, Adleman NE, Dienes K, Simeonova DI, Menon V, Reiss A. Anomalous prefrontal-subcortical activation in familial pediatric bipolar disorder: a functional magnetic resonance imaging investigation. Arch Gen Psychiatry. 2004;61(8):781-92.

24. Kim P, Thomas LA, Rosen BH, Moscicki AM, Brotman MA, Zarate Jr CA, et al. Differing amygdala responses to facial expressions in children and adults with bipolar disorder. Am J Psychiatry. 2012;169(6):642-9.

25. Pavuluri MN, Schenkel LS, Aryal S, Harral E, Hill SK S, Herbener ES, et al. Neurocognitive function in unmedicated manic and medicated euthymic pediatric bipolar patients. Am J Psychiatr. 2006;163:286-93.

26. Pavuluri M, May A. I feel, therefore, I am: the insula and its role in human emotion, cognition and the sensorymotor system. AIMS Neurosci. 2015;2(1):18-27.

27. Pavuluri MN, Passarotti AM, Harral EM, Sweeney JA. An fMRI study of the neural correlates of incidental versus directed emotion processing in pediatric bipolar disorder. J Am Acad Child Adolesc Psychiatry. 2009;48(3):308-19.

28. Pavuluri MN, Passarotti AM, Mohammed T, Carbray JA, Sweeney JA. Enhanced working memory after lamotrigine treatment in pediatric bipolar disorder. Bipolar Disord. 2010;12(2):213-20.

29. Rich BA, Holroyd T, Carver FW, Onelio LM, Mendoza $\mathrm{JK}$, Cornwell BR, et al. A preliminary study of the neural mechanisms of frustration in pediatric bipolar disorder using magnetoencephalography. Depress Anxiety. 2010;27(3):276-86

30. Patino LR, Adler CM, Mills NP, Strakowski SM, Fleck DE, Welge JA, et al. Conflict monitoring and adaptation in individuals at familial risk for developing bipolar disorder. Bipolar Disord. 2013;15(3):264.

31. Gorrindo T, Blair RJ, Budhani S, Dickstein DP, Pine DS, Leibenluft E. Deficits on a probabilistic responsereversal task in patients with pediatric bipolar disorder. Am J Psychiatry. 2005;162(10):1975-7.

32. Joseph MF, Frazier TW, Youngstrom EA, Soares JC. A quantitative and qualitative review of neurocognitive performance in pediatric bipolar disorder.

33. Jacobs RH, Pavuluri MN, Schenkel LS, Palmer A, Shah $K$, Vemuri D, et al. Negative emotion impacts memory for verbal discourse in pediatric bipolar disorder. Bipolar Disord. 2011;13(3):287-93.

34.• Passarotti AM. Neurocognitive Models of Evolving Bipolar Disorder in Youth. In: Strakowski SM, DelBello 
MP, Adler CM, editors. 'Progression of Bipolar Disorder in Youth: Presentation, Treatment and Neurobiology'. NY: Oxford University Press; 2014.

Excellent overview of neurocognitive problems in pediatric bipolar disorder.

35. Dickstein DP, Gorrostieta C, Ombao H, Goldberg LD, Brazel AC, Gable CJ, et al. Fronto-temporal spontaneous resting state functional connectivity in pediatric bipolar disorder. Biol Psychiatry. 2010;68(9):839-46.

36. Strakowski SM, Fleck DE, DelBello MP, Adler CM, Shear PK, Kotwal R, et al. Impulsivity across the course of bipolar disorder. Bipolar Disord. 2010;12(3):285-97.

37. Pavuluri MN, Oconnor-Marlow M, Harral E, Sweeney JA. Role of the affective circuitry during facial emotion processing in pediatric bipolar disorder. Biol Psychiatry. 2007;62:158-67.

38. Whitney J, Joormann J, Gotlib IH, Kelley RG, Acquaye $\mathrm{T}$, Howe $\mathrm{M}$, et al. Information processing in adolescents with bipolar I disorder. J Child Psychol Psychiatry. 2012;53(9):937-45.

39. Passarotti AM, Fitzgerald JM, Sweeney JA, Pavuluri MN. Negative emotion interference during a synonym matching task in pediatric bipolar disorder with and without attention deficit hyperactivity disorder. J Int Neuropsychol Soc 2013. 2013;19(5):601-12.

40. Brotman MA, Rich BA, Schmajuk M, Reising M, Monk CS, Dickstein DP, et al. Attention bias to threat faces in children with bipolar disorder and comorbid lifetime anxiety disorders. Biol Psychiatry. 2007;61(6):819-21.

41. Leibenluft E, Rich BA, Vinton DT, Nelson EE, Fromm SJ, Berghorst LH, et al. Neural circuitry engaged during unsuccessful motor inhibition in pediatric bipolar disorder. Am J Psychiatry. 2007;164(1):52-60.

42. Deveney CM, Connolly ME, Jenkins SE, Kim P, Fromm SJ, Pine DS, et al. Neural recruitment during failed motor inhibition differentiates youths with bipolar disorder and severe mood dysregulation. Biol Psychol. 2012;89(1):148-55.

43. Weathers JD, Stringaris A, Deveney CM, Brotman MA, Zarate Jr CA, Connolly ME, et al. A developmental study of the neural circuitry mediating motor inhibition in bipolar disorder. Am J Psychiatry.

2012;169(6):633-41.

44. Passarotti AM, Sweeney JA, Pavuluri MN. Frontolimbic dysfunction in mania pre-treatment and persistent amygdala over-activity post-treatment in pediatric bipolar disorder. Psychopharmacology.

2011;216(4):485-99.

45. Workshop on the Cognitive Systems Domain, 2011 October 23-25, 2011; Rockville, MD: Research domain criteria (RDoC) project.

46. Cerullo MA, Adler CM, Lamy M, Eliassen JC, Fleck DE, Strakowski SM, et al. Differential brain activation during response inhibition in bipolar and attention-deficit hyperactivity disorders. Early Interv Psychiatry. 2009;3(3):189-97.

47. Pavuluri MN, Birmaher B, Naylor MW. Pediatric bipolar disorder: a review of the past 10 years. J Am Acad Child Adolesc Psychiatry. 2005;44(9):846-71.
48. Adler CM, DelBello MP, Strakowski SM. Brain network dysfunction in bipolar disorder. CNS Spectr. 2006;11(4):312-20.

quiz 23-4.

49. Passarotti AM, Sweeney JA, Pavuluri MN. Emotion processing influences working memory circuits in pediatric bipolar disorder and attention-deficit/hyperactivity disorder. J Am Acad Child Adolesc Psychiatry. 2010;49(10):1064-80.

50. Passarotti AM, Ellis J, Wegbreit E, Stevens M, Pavuluri $\mathrm{MN}$. Reduced functional connectivity of prefrontal regions and amygdala within affect and working memory networks in pediatric bipolar disorder. Brain Connect. 2012;2(6):320-34.

51. May JC, Delgado MR, Dahl RE, Stenger VA, Ryan ND, Fiez JA, et al. Event-related functional magnetic resonance imaging of reward-related brain circuitry in children and adolescents. Biol Psychiatry. 2004;55(4):359-66.

52. Bjork JM, Hommer DW. Anticipating instrumentally obtained and passively-received rewards: a factorial fMRI investigation. Behav Brain Res.

2007;177(1):165-70.

53. Schenkel LS, Marlow-O'Connor M, Moss M, Sweeney JA, Pavuluri MN. Theory of mind and social inference in children and adolescents with bipolar disorder. Psychol Med. 2008;38(6):791-800.

54. Schenkel LS, Chamberlain TF, Towne TL. Impaired theory of mind and psychosocial functioning among pediatric patients with type I versus type II bipolar disorder. Psychiatry Res. 2014;215(3):740-6.

55. Whitney J, Howe M, Shoemaker V, Li S, Marie Sanders E, Dijamco C, et al. Socio-emotional processing and functioning of youth at high risk for bipolar disorder. J Affect Disord. 2013;148(1):112-7. doi:10.1016/j.jad. 2012.08.016.

56. Schenkel LS, Passarotti AM, Sweeney JA, Pavuluri MN. Negative emotion impairs working memory in pediatric patients with bipolar disorder type I. Psychol Med. 2012;42(12):2567-77.

57. Passarotti AM, Sweeney JA, Pavuluri MN. Neural correlates of response inhibition in pediatric bipolar disorder and attention deficit hyperactivity disorder. Psychiatry Res. 2010;181(1):36-43.

58. Pavuluri ACNP Psychopharm.

59. Mayanil T, Wegbreit E, Fitzgerald J, Pavuluri MN. Emerging biosignature of brain function and intervention in pediatric bipolar disorder. Minerva Pediatr. 2011;63(3):183-200.

60. Pavuluri MN, Passarotti AM, Harral EM, Sweeney JA. Enhanced prefrontal function with pharmacotherapy on a response inhibition task in adolescent bipolar disorder. J Clin Psychiatry. 2010;71(11):1526-34.

61. Chang KD, Wagner C, Garrett A, Howe M, Reiss A. A preliminary functional magnetic resonance imaging study of prefrontal-amygdalar activation changes in adolescents with bipolar depression treated with lamotrigine. Bipolar Disord. 2008;10:426-31. 
62. Pavuluri MN, Ellis J, Wegbreit E, Passarotti A, Stevens M. Pharmacotherapy impacts functional connectivity among affective circuits during response inhibition in pediatric mania. Behav Brain Res. 2012;226(2):493503.

63. Pavuluri MN, Passarotti AM, Lu LH, Carbray JA, Sweeney JA. Double-blind randomized trial of risperidone versus divalproex in pediatric bipolar disorder: fMRI outcomes. Psychiatry Res Neuroimaging. 2011;193(1):28-37.

64. Wegbreit E, Ellis J, Nandam A, Fitzgerald J, Passarotti A, Pavuluri MN, et al. Amygdala functional connectivity predicts pharmacotherapy outcome in pediatric bipolar disorder. Brain Connect. 2011;1(5):411-22.

65. Yang H, Lu L, Wu M, Stevens M, Wegbreit E, Fitzgerald $\mathrm{J}$, et al. Time course of recovery showing initial prefrontal cortex changes at 16 weeks times. J Affect Disord. 2013;13:123-7.

66. DelBello MP, Soutullo CA, Hendricks W, Niemeier RT, McElroy SL, Strakowski SM. Prior stimulant treatment in adolescents with bipolar disorder: association with age at onset. Bipolar Disord. 2001;3(2):53-7.

67. Bhowmik D, Aparasu RR, Rajan SS, Sherer JT, OchoaPerez M, Chen H. Risk of manic switch associated with antidepressant therapy in pediatric bipolar depression. J Child Adolesc Psychopharmacol. 2014;24(10):551-61.

68. http://www.nice.org.uk/guidance/conditions-anddiseases/mental-health-and-behavioural-conditions/ bipolar-disorder, Retrieved on May 29, 2015.

69. Mattei C, Rapagnani MP, Stahl SM. Ziprasidone hydrocloride: what role in the management of schizophrenia? J Cent Nerv Syst Dis. 2011;3:1-16.

70. Baldessarini RJ, Tohen M, Tondo L. Maintenance treatment in bipolar disorder. Arch Gen Psychiatry. 2000;57(5):490-2.

71. Tondo L, Baldessarini RJ. Reduced suicide risk during lithium maintenance treatment. J Clin Psychiatry. 2000;61 Suppl 9:97-104.

72. Pavuluri MN, Lee M-S, Pandey G. Lithium response viewed as a biomarker to predict developmental psychopathology in bipolar offspring. Bipolar Disord. 2014. doi:10.1111/bdi.12280.

73. Pavuluri MN, Janicak PG. Handbook of psychopharmacotherapy: a life-span approach / Edition 2. 2008. Lippincott Williams \& Wilkins.

74. Detke H, DelBello MP, Landry J, Math M, Usher R. Olanzapine/fluoxetine combination in children and adolescents with bipolar i depression: a randomized, double-blind, placebo-controlled trial. J Am Acad Child Adolesc Psychiatry. 2015;54(3):217-24.

75.• West AE, Weinstein SM, Peters AT, Katz A, Henry DB, Cruz R, et al. Child and family-focused cognitive-behavioral therapy for pediatric bipolar disorder: A randomized clinical trial. J Am Acad Child Adolesc Psychiatry. 2014;53(11):1168-78.

Latest data on emotion regulation that is built on neuroscience formulation.

76. West AE, Pavuluri MN. Maintenance model of integrated psychosocial treatment in pediatric bipolar disorder. J Am Acad Child Adolesc Psychiatry. 2007;46(2):205-12.

77. Goldstein TR, Fersch-Podrat RK, Rivera M, et al. Dialectical behavior therapy (DBT) for adolescents with bipolar disorder: results from a pilot randomized trial. J Child Adolesc Psychopharmacol. 2014.

78. MacPherson HA, Cheavens JS, Fristad MA. Dialectical behavior therapy for adolescents: theory, treatment adaptations, and empirical outcomes. Clin Child Fam Psychol Rev. 2013;16(1):59-80.

Good model for emotion regulation, drawing from the complexity of treatment for immediate gratification and emotion regulation.

79. Miklowitz DJ, Otto MW, Frank E, et al. Psychosocial treatments for bipolar depression: a 1-year randomized trial from the Systematic Treatment Enhancement Program. Arch Gen Psychiatry. 2007;64(4):419-26.

A good overview of intervention model for emotion regulation. 